\title{
Recipe Recommendation Based on Ingredients using Machine Learning
}

\author{
Ms. Soundarya Desai ${ }^{1}$, Ms. Pooja Patil ${ }^{2}$, Mr. Pratik Shinde ${ }^{3}$, Mr. Azhar Sayyed ${ }^{4}$, Prof. Rohini Bhosale ${ }^{5}$ \\ Student, Comp Dept, KJEI's Trinity College of Engineering and Research Pisoli, Pune, India ${ }^{1,2,3,4}$ \\ Guide, Comp Dept, KJEI's Trinity College of Engineering and Research Pisoli, Pune, India ${ }^{5}$
}

\begin{abstract}
Thinking of what to cook is also a difficult problem. To attract children liking, parent need to exchange the menu every day. Parents not only think to what recipe to changes, they also need to consider the nutrition that their children taken. Besides that, some people will forget buy ingredients to stock in their kitchen. This will become a problem when they want to prepare meal within short time. It is difficult to think what to cook with limited ingredient that in the kitchen Many people often cook a dish with a cooking recipe on Websites and magazines. The listed ingredients in the recipe sometimes cannot be prepared. This paper propose a recommendation system for different ingredients. The recommendation ingredients based on co-occurrence frequency of ingredients on recipe database and ingredient category stored in a cooking ontology. For object detection open CV is used which is java based. Basically we are using this platform for feature extraction of ingredients. Grey scale of the image is calculated, further histogram is generated which helps in identification of the food items.
\end{abstract}

Keywords: Recipe, Ingredient, User, Interest

\section{INTRODUCTION}

It is natural to think that couples who work at a company or a person who lives by her/himself want to cook food for themselves as quickly and easily as possible when they are busy. However, to keep having the same food they can easily cook fed them up, therefore, it should be preferable for them to be recommended a variety of food that they can cook "easily". Currently, there are so many Web sites for cooking recipes, and there are also recipes regarded as "easy" to cook. However, those recipes are not estimated as "easy" by taking user's conditions into account. Therefore, in this system, we aim to develop a method to recommend "easy" cooking recipes by analyzing the content of recipes and considering user's conditions and then develop a recommendation system with the proposed method. Recommender systems are very helpful when the recommended item is something that the user has not seen in the past any. For example, popular movies of a preferred genre would not mostly to be novel to the user. Repeated recommendation of popular items can also lead to decrease in sales diversity.

\section{LITERATURE SURVEY}

A Recipe Recommendation System Based on Regional Flavor Similarity Lin-rong GUO, Shi-zhong YUAN* , Xue-hui MAO and Yi-ning GU School of Computer Engineering and Science, Shanghai University, Shanghai 200444, China.[1]

One of the factors that affect food choice is flavor preferences. Although the flavors of cuisines vary from one region to another, there are flavor similarities among the cuisines from geographically adjacent regions. This paper presents a recipe recommendation system to recommend a set of dishes from the various Chinese regional cuisines for a certain flavor preference in terms of flavor similarity. The flavor similarities among the cuisines are determined using our previously developed algorithms. First, the TF-IDF (Term Frequency-Inverse Document Frequency) algorithm is used to calculate the ingredient preferences of the regional cuisines, on the basis of which, each dish of the regional cuisines is given a score. Then, we use the cosine similarity to measure the flavor similarities among the regional cuisines. Finally, the Tidal-Trust algorithm is employed to choose the dishes with the most similar flavors and recommend them to the user. The results of the questionnaire evaluation for the system show the recommendations from the system are reasonable and acceptable from professional chefs' point of view.

"Constraint based recipe recommendation using forward checking algorithm ",Kirti R. Pawar ; Tushar Ghorpade ; Rajashree Shedge 2016 International Conference on Advances in Computing, Communications and Informatics (ICACCI)[2] 
With the advanced research in medical science and technology, quality of our life has improved, also life span has been increased. With life style, diet habits has changed and work pressure increased which resulted in number of diseases, such as diabetes, Blood Pressure, heart problem etc. These diseases can be controlled to certain extent by avoiding uneven and inappropriate diet. Hence it is important to understand good diet and intake habits. Our objective is to recommend recipes to maintain health condition for people with disease and without disease which will satisfy the user needs. To present the recommendations, recommended system uses user's profile, favourite items and recipe details. This explicit knowledge satisfies the constraints for the recommendation. In this paper we propose a recommendation system for recipe using constraint knowledge based recommendation method and forward checking algorithm. The proposed system suggests recipe for diabetes disease which provides improved precision and recall. This depicts that proposed system is efficient than existing system. The reason to select this method is that it does not have ramp-up problem. The constraints which satisfy user's requirement will be the good recipe recommendation.

Implementation of a Goal-Oriented Recipe Recommendation System Providing Nutrition Information",Tsuguya Ueta ; Masashi Iwakami ; Takayuki Ito, 2011 International Conference on Technologies and Applications of Artificial Intelligence[3]

Recently, the number of people who use recipe sites is increasing. Since professional knowledge of nutrition is necessary to decide daily recipes for improving health, it may be difficult for general users who do not have such knowledge to search for the right recipes. In this paper, we propose a goal-oriented recipe recommendation system that utilizes information about nutrition on the Internet. Our system enables users without knowledge about nutrition to search easily for recipes with natural language to improve specific health conditions. The natural language includes 'I want to cure my acne 'and 'I want to recover from my fatigue '. We compared the results of our system to the results we obtained by calculating the nutrient information manually. Evaluation was done on 1000 dishes. We measured the effectiveness of the system using F-Measure and the average F-measure was 0.64 respectively.

“An overview of recommender systems in the healthy food domain." Thi Ngoc Trang Tran Müslüm Atas, Alexander Felfernig.[4]

Recently, food recommender systems have received increasing attention due to their relevance for healthy living. Most existing studies on the food domain focus on recommendations that suggest proper food items for individual users on the basis of considering their preferences or health problems. These systems also provide functionalities to keep track of nutritional consumption as well as to persuade users to change their eating behavior in positive ways. Also, group recommendation functionalities are very useful in the food domain, especially when a group of users wants to have a dinner together at home or have a birthday party in a restaurant. Such scenarios create many challenges for food recommender systems since the preferences of all group members have to be taken into account in an adequate fashion. In this paper, we present an overview of recommendation techniques for individuals and groups in the healthy food domain. In addition, we analyze the existing state-of-the-art in food recommender systems and discuss research challenges related to the development of future food recommendation technologies.

\section{PROPOSE SYSTEM}

We propose web based application which construct recommendation system by adding more heterogeneous information of recipes like cuisines, preparation direction, dietary etc. In this system there are Three main module

1. Android app(User):-In this user get registered with system and add images .

2. Web App(User):-In this user can add recipe, add view and comments on recipe and search recipe they want.

3. Admin:-Admin is responsible for adding user and managing other module.

\section{SYSTEM ARCHITECTURE}

Following diagram is our system's architecture diagram:

- User Module: In user module user have to get registered with system with unique id and password. After successfully registeration user can login with system.

- Login: After successful registeration, user can login and get authority to access system of personal travelling scheduler.

- Admin: Admin in this system is responsible for adding user , authentication and managing other modules ,adding ingredient ,adding recipe and managing 
Vol. 8, Issue 3, March 2019

- Android Application (User): User in android application can get registered with system with unique id and login. After get authentication by admin user can access system and can upload images.

- Web Application(User): User in web application can view/browse the recipe he/she want and can post new recipe(not compulsory). User can also review and comments on recipe on the system as he want.

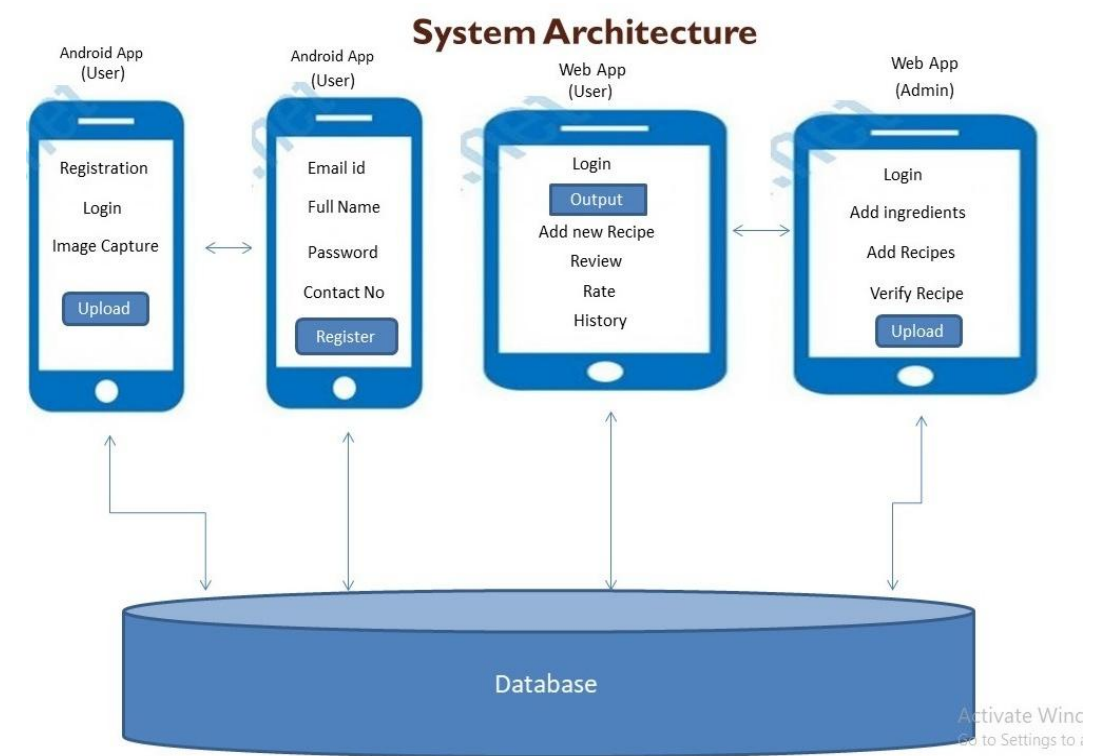

Figure 1: system architecture

\section{METHODOLOGIES}

\section{Mathematical Model}

Let_S be the | universal final set. This will include user, resources, system.

$\mathrm{S}=\{\ldots \ldots \ldots \ldots\}$

Identify the inputs as I

$\mathrm{I}=\{\mathrm{I}\}$

$\mathrm{F}=\{\mathrm{I} 1, \mathrm{I} 2, \mathrm{I} 3, \mathrm{I} 4 \ldots \mid=\mathrm{I}$ 'image by user $\}$

Identify the outputs as $\mathrm{O}$

$\mathrm{O}=\{\mathrm{R}\}$

$\mathrm{R}=\{$ Recipe ... $\mid=\mathrm{R}$ 'given recommended recipe $\}$

Identify the functions as $=F^{6}$

$\mathrm{S}=\{\ldots$

$\mathrm{F}=\{\mathrm{F} 1(), \mathrm{F} 2(), \mathrm{F} 3(), \mathrm{F} 4(), \mathrm{F} 5(), \mathrm{F} 6(), \mathrm{F} 7()\}$

$\mathrm{F} 1(\mathrm{I})=\operatorname{login}$

$\mathrm{F} 2(\mathrm{~V})=$ upload image

F3 $(\mathrm{V})=$ fileted content

$\mathrm{F} 4(\mathrm{~V})=$ identify ingredients

$\mathrm{F} 5(\mathrm{~V})=$ recommend recipe

$\mathrm{F} 6(\mathrm{~V})=$ rate

Deterministic data $=$ same output from a given starting condition and return the same result any time

Non deterministic data $=$ result will vary every time for given input

Success condition $=$ According to proper inputs, recommend recipe

failure condition $=$ Wrong inputs and ambiguity in images

\section{CONCLUSION}

The proposed system is web based application .The project is Food Recommendation System by using Content Based Filtering Algorithm. The data set are collected by web scraping which is pre-processed on the basis attributes. The data are then used to model the system. This application is extremely handy and useful for cooking variety of recipe with minimum search effort from internet. This app also provides ability to user to create recipe and save it for later reference. It will help people to save their time and energy in finding recipes for daily routine as well as for special occasions 


\section{FUTURE WORK}

- For future work, we plan to improve the system in terms of object recognition, recipe recommendation and the system user interface.

- $\quad$ Regarding object recognition, we would like to achieve $90 \%$ classification rate within the top six candidates for the 100 category food ingredients by adding other image features and segmenting food ingredient regions from background regions.

- Regarding recipe recommendation, we plan to implement recipe search considering combination of multiple food ingredients, nutrition and budgets.

\section{RESUlt}

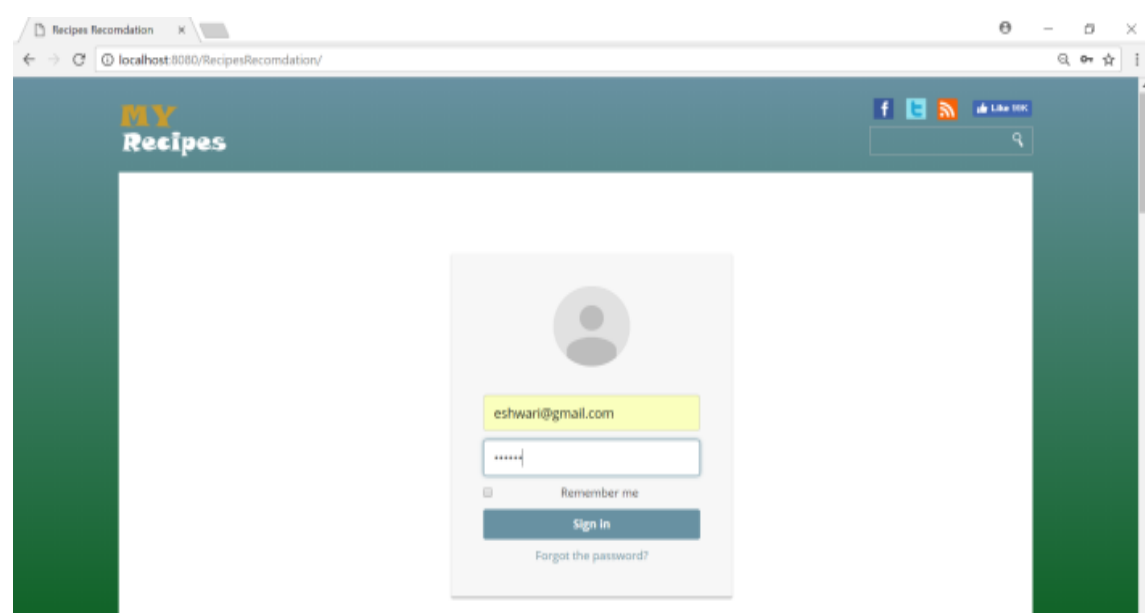

This figure shows the registration and login page of the user and admin module.

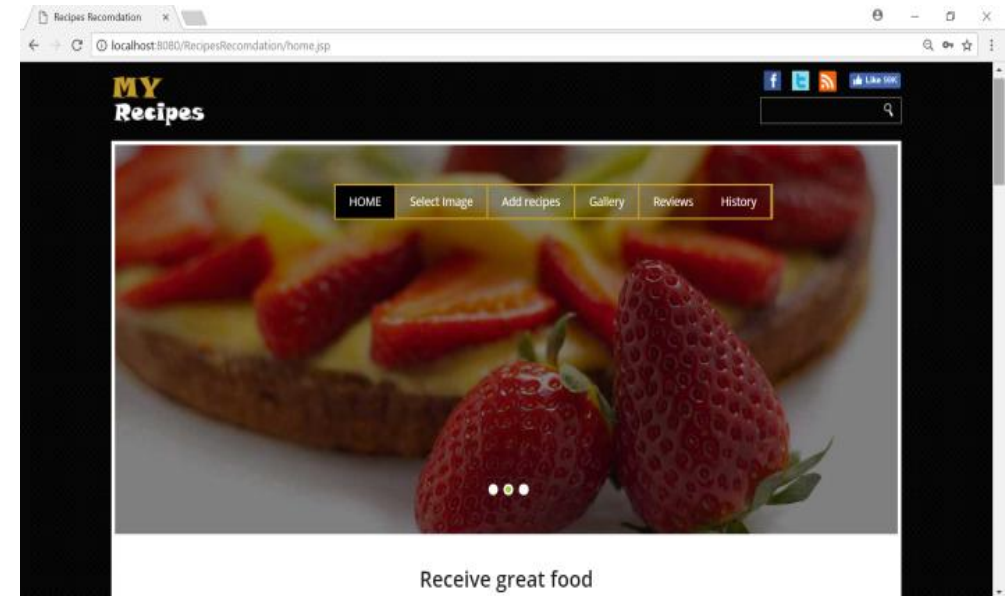

This figure shows the dashboard of system.

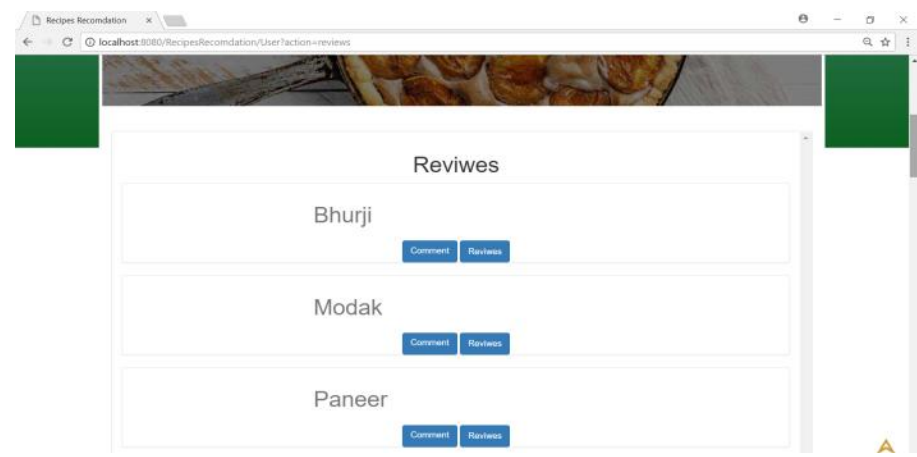

After completeing all process we will get to know the reviews of the recipes. 
International Journal of Advanced Research in Computer and Communication Engineering

Vol. 8, Issue 3, March 2019

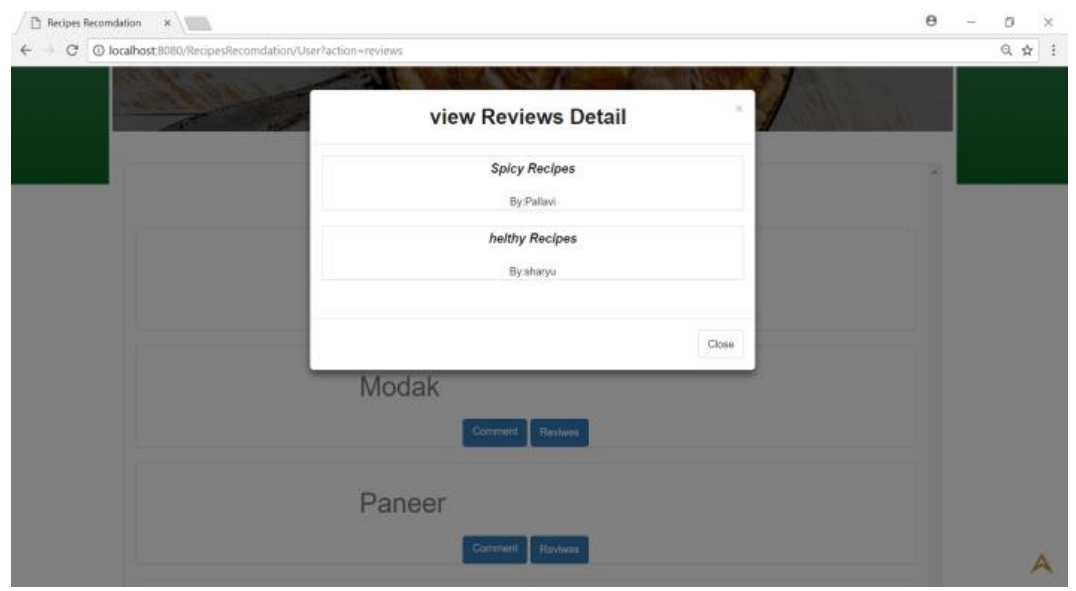

This figure shows the review details of the particular recipe.

\section{REFERRENCES}

[1]. A Scalable recipe recommendation system for mobile application ",Zhengxian Li, Jinlong Hu, Jiazhao Shen, Yong Xu

[2]. Constraint based recipe recommendation using forward checking algorithm “,Kirti R. Pawar ; Tushar Ghorpade ; Rajashree Shedge 2016 International Conference on Advances in Computing, Communications and Informatics (ICACCI)

[3]. Implementation of a Goal-Oriented Recipe Recommendation System Providing Nutrition Information", Tsuguya Ueta; Masashi Iwakami ; Takayuki Ito, 2011 International Conference on Technologies and Applications of Artificial Intelligence

[4]. Distributed Representation of Dish Names in Food Related Web Services for Associative Search and Nutrition Estimation : Yuguan Xing, Sosuke Amano, 2017

[5]. Jingjing Chen, Chong-Wah Ngo, "Deep-based Ingredient Recognition for Cooking Recipe Retrieval”, 2017.

[6]. Jay Baxter, "Food Recognition using Ingredient-Level Features", 2017.

[7]. Recommendation System for Alternative-ingredients Based on Co-occurrence Relation on Recipe Database and the Ingredient Category : Naoki SHINO, 2016

[8]. Food recognition and recipe analysis: integrating visual content, context and external knowledge : Luis Herranz, 2016 